\title{
Measurement of the energy spectrum of ultra-high energy cosmic rays using the Pierre Auger Observatory
}

\author{
Valerio Verzi ${ }^{* a}$ for the Pierre Auger Collaboration ${ }^{b \dagger}$ \\ ${ }^{a}$ Sezione INFN Roma "Tor Vergata", via della Ricerca Scientifica 1, 00133 Roma, Italy \\ ${ }^{b}$ Observatorio Pierre Auger, Av. San Martín Norte 304, 5613 Malargüe, Argentina \\ E-mail: auger_spokespersons@ @ fnal.gov \\ Full author list: http://www.auger.org/archive/authors_icrc_2019.html
}

\begin{abstract}
The energy spectrum of ultra-high energy cosmic rays measured using the Pierre Auger Observatory is presented. The measurements benefit from the huge exposure of approximately 80000 $\mathrm{km}^{2} \mathrm{sr}$ yr achieved in 14 years of data taking with a surface-detector array that extends over 3000 $\mathrm{km}^{2}$ having 1600 detectors on a $1500 \mathrm{~m}$ spacing, and from the almost-calorimetric estimation of the energy scale provided by the fluorescence detector. In this contribution, we address recent improvements in the measured spectrum at energies above $3 \mathrm{EeV}$ using events with zenith angles less than $60^{\circ}$. These improvements concern the estimation of the shower energy and its resolution. Further, we report on updates of the energy spectra derived from other independent and complementary data sets, namely from showers with larger zenith angles, those detected by a smaller and denser array with $750 \mathrm{~m}$ spacing, and those detected by the fluorescence detector, together with the recent extension of the flux measurements to lower energies using atmospheric Cherenkov radiation.
\end{abstract}

36th International Cosmic Ray Conference -ICRC2019-

July 24th - August 1st, 2019

Madison, WI, U.S.A.

\footnotetext{
* Speaker.

${ }^{\dagger}$ for collaboration list see PoS(ICRC2019)1177
} 


\section{Introduction}

The Pierre Auger Observatory [1] is located in a region called Pampa Amarilla, near the small town of Malarguie in the province of Mendoza (Argentina) at a latitude of about $35.2^{\circ} \mathrm{S}$ and an altitude of $1400 \mathrm{~m}$ above sea level. The Observatory, completed in 2008, is a hybrid system, a combination of a large surface detector (SD) and a fluorescence detector (FD).

The SD comprises 1660 water-Cherenkov detectors (WCD) laid out on a $1500 \mathrm{~m}$ triangular grid, covering an area of about $3000 \mathrm{~km}^{2}$, and an additional 61 detectors covering $23.5 \mathrm{~km}^{2}$ on a $750 \mathrm{~m}$ grid. The FD consists of $4 \times 6$ telescopes placed in four locations on the perimeter of the site (also called eyes) that detect the fluorescence light emitted during the shower development. Each telescope has a field of view of $30^{\circ} \times 30^{\circ}$ with a minimum elevation of $1.5^{\circ}$ above the horizon. Three additional telescopes, the High Elevation Auger Telescopes (HEAT), cover an elevation up to $60^{\circ}$ to detect low-energy showers in coincidence with the $750 \mathrm{~m}$ array. The FD may operate only in clear moonless nights and therefore with an on-time of about $13 \%$.

The main advantage of a hybrid system is that the energy scale of the Observatory can be set with the FD measurements that provide an almost calorimetric estimate of the shower energy. This allows us to measure the energy spectrum with the high efficiency of the SD and with an energy estimation which is largely independent of air shower simulations and of assumptions on hadronic interaction models.

In this contribution we present the energy spectrum measured at the Pierre Auger Observatory using an exposure of about $80000 \mathrm{~km}^{2}$ sr yr. First we describe the recent improvements in the spectrum measured with the $1500 \mathrm{~m}$ array using events with zenith angles $(\theta)$ less than $60^{\circ}$. We then report on updates of the energy spectra derived from other independent and complementary data sets. In comparison to our previous publication [2], the energy threshold above which we measure the spectrum is lowered by one decade down to $10^{16.5} \mathrm{eV}$. We will present the spectral features in the full energy range, from $10^{16.5} \mathrm{eV}$ up to the suppression of the flux at the highest energies.

\section{The energy spectrum from the $1500 \mathrm{~m}$ array using events with $\theta<60^{\circ}$}

The reconstruction of events detected by the $1500 \mathrm{~m}$ array with zenith angles less than $60^{\circ}$ is described in [3]. The shower size and core position are estimated by fitting to the data a modified Nishimura-Kamata-Greisen lateral distribution function (LDF) with slope parameters determined from data which are a function of the shower size and zenith angle. The shower size is the signal at $1000 \mathrm{~m}$ from the core in the plane of the shower front $(S(1000)) . S(1000)$ is the optimal energy estimator for a grid spacing of $1500 \mathrm{~m}$ because it minimises the uncertainties of the signal due to limited knowledge of the LDF in individual events [4]. $S(1000)$ is measured in units of vertical equivalent muon (VEM). 1 VEM corresponds to the signal released by a muon traversing the tank vertically and it is measured for each WCD every $60 \mathrm{~s}$ [1].

For a given energy, the value of $S(1000)$ decreases with the zenith angle because of the increasing atmospheric depth crossed by the shower. Given the highly isotropic flux, the shape of the attenuation curve can be inferred from data using the Constant Intensity Cut (CIC) method [5]. The curve is parameterised with a third degree polynomial in terms of the variable $x=\cos ^{2} \theta-\cos ^{2} 38^{\circ}$, 
where $S(1000)=S_{38}\left(1+a x+b x^{2}+c x^{3}\right) . \quad S_{38}$ is the zenith-angle independent energy estimator and can be thought of as the signal, $S(1000)$, that the shower would have produced at a zenith angle of $38^{\circ}$. In our previous publication [2] the coefficients $a, b$ and $c$ were calculated at a fixed intensity threshold number of events per steradian above a given $S(1000)$ threshold). In figure 1 we show how the shape of the attenuation curves are slightly different for different intensity thresholds. Thus, to obtain a more precise energy estimator, for the measurements presented in this paper, the CIC is calculated at different thresholds. In practice, the energy dependence of the CIC curve is accounted for by expressing the coefficients $a, b$ and $c$ with a second degree polynomial in the variable $k=\log _{10}\left(S_{38} / 40\right.$ VEM $)$, i.e. $y=$ $\sum_{l}^{[0,2]} y_{l} k^{l}$. The value of the coefficients $\left(y_{0}, y_{1}, y_{2}\right)$ are: $(0.952,0.0587,-0.370)$

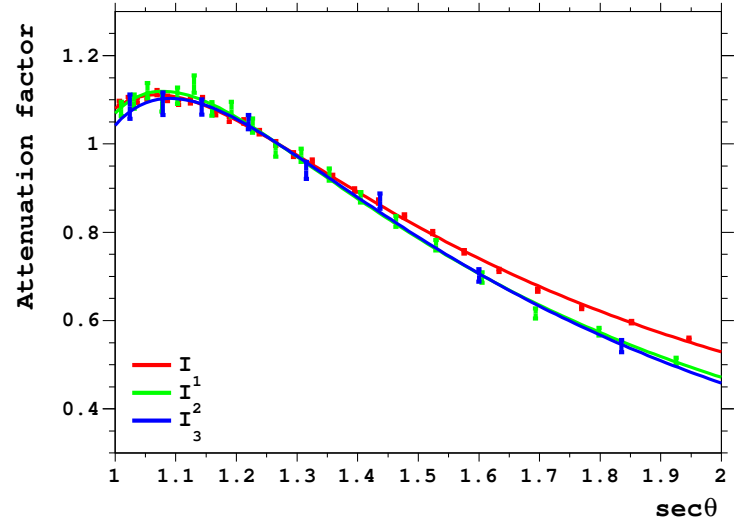

Figure 1: Attenuation curves as a function of $\sec \theta$ normalised to 1 for $\theta=38^{\circ}$ for the three different intensity thresholds that correspond approximatively to the energies $3 \mathrm{EeV}\left(\mathrm{I}_{1}\right), 8 \mathrm{EeV}\left(\mathrm{I}_{2}\right)$ and $20 \mathrm{EeV}\left(\mathrm{I}_{3}\right)$. for $a,(-1.636,-0.425,0.087)$ for $b$ and $(-0.978,-0.041,1.335)$ for $c$. The parameterisation is valid for $S_{38}$ between 15 VEM and 120 VEM. Outside this range, we use the coefficients calculated on the boundaries of the validity range.

The calibration of $S_{38}$ against the calorimetric energy $E_{\mathrm{FD}}$ is obtained by analysing the so called hybrid events, that are a subset of SD events where the FD was triggered independently. The reconstruction of the FD events is described in [1] and provides an estimation of the calorimetric energy of the showers $\left(E_{\mathrm{cal}}\right)$. The total shower energy $\left(E_{\mathrm{FD}}\right)$ is obtained by adding to $E_{\text {cal }}$ an invisible energy correction that accounts for the energy carried into the ground by highenergy muons and neutrinos. This correction is estimated by exploiting the sensitivity of the WCDs to muons with an analysis that minimises the uncertainties arising from the hadronic interaction models and the primary mass composition [6]. The hybrid events are selected to guarantee a precise estimation of the FD energies and to minimise biases from the mass distribution of the cosmic rays introduced by the field of view of the FD telescopes [6]. The calibration is performed by selecting events with $E_{\mathrm{FD}}>3 \times 10^{18} \mathrm{eV}$ to guarantee a nearly $100 \%$ trigger efficiency of the SD array [7]. The correlation between the FD energies and $S_{38}$ of 3338 events selected from the data collected between 1 January 2004 to 31 December 2017 is shown in figure 2. The correlation is well described by a simple power-law relationship $E=A S_{38}^{B}$ where the two parameters $A$ and $B$ are fitted to the data. For the fit we use a maximum-likelihood method where the probability density function is given by a bootstrap estimate of the energy distribution of the selected events and where the uncertainties in $S_{38}$ and FD energy [8] are evaluated on an event-by-event basis. The uncertainties in $S_{38}$ are defined by considering the error from the $S(1000)$ fit [3] and shower-to-shower fluctuations (they amount to about $13 \%-7 \%$, lower at higher energies). The latter are estimated by subtracting from the total SD energy resolution (which will be presented later) the errors from the $S(1000)$ fit. The best fit parameters are 
$A=(0.186 \pm 0.003) \mathrm{EeV}$ and $B=1.031 \pm 0.004$ and the correlation coefficient between them is $\rho=$ -0.98 . The resulting calibration curve is shown as the red line in figure 2 . The highest-energy event is detected by all four FD eyes. Its energy is $(8.5 \pm 0.4) \times 10^{19} \mathrm{eV}$, obtained from a weighted average of the four calorimetric energies and using the resulting energy to evaluate the invisible energy correction [6]. The corresponding SD energy obtained from $S_{38}$ using the calibration parameters is $(7.9 \pm 0.6) \times 10^{19} \mathrm{eV}$, in good agreement with the FD energy.

The parameters $A$ and $B$ define the energy scale of the $1500 \mathrm{~m}$ array and are used to estimate the energy for the bulk of $\mathrm{SD}$ events. The systematic uncertainty in the energy scale is $14 \%$ [9]. It is approximately constant with energy, being dominated by the uncertainty in the absolute calibration of the FD telescopes, and benefits from the high precision measurement

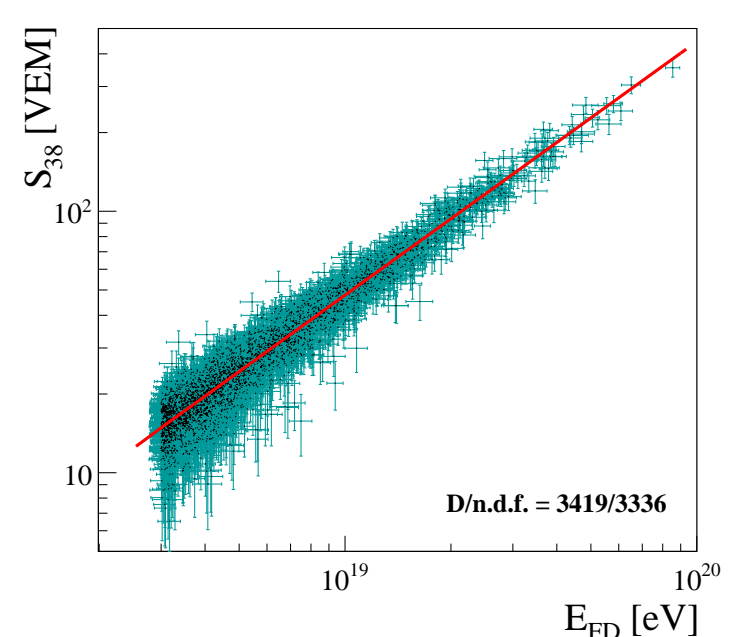

Figure 2: Correlation between the FD energies and $S_{38}$. Each event is shown with a point together with its individual uncertainties. The line is the best fit calibration curve. of the fluorescence yield made by the AIRFLY experiment [10]. After the major revision of the energy scale that was presented in 2013 [9], the Auger Collaboration has made several checks and improvements in the estimation of the FD energies. The results of these activities are reported in [8] and no effect has been discovered that contradicts the estimation of the systematic uncertainties addressed in [9].

The estimation of the differential energy spectrum is done by counting the number of SD events $N_{i}$ in differential bins centered at energy $E_{i}$ with equal-size width in decimal logarithm $\Delta \log E_{i}=0.1$ :

$$
J=f\left(E_{i}\right) J_{\text {raw }}=f\left(E_{i}\right) \frac{N_{i}}{\mathscr{E} \Delta E_{i}}
$$

where $\mathscr{E}$ is the exposure, $f\left(E_{i}\right)$ accounts for resolution effects responsible for a bin-to-bin event migration and $J_{\text {raw }}$ is the estimation of the spectrum neglecting the resolution effects. The spectrum $J$ is estimated by selecting events in which the WCD with the highest signal is enclosed in a hexagon of six active stations and requiring that the events have an energy larger than $10^{18.4} \mathrm{eV}$ and zenith angle less than $60^{\circ}$. In this way the trigger efficiency is larger than $97 \%$ and the calculation of the exposure reduces to a geometrical calculation plus knowledge of the live-time of the array [7]. For the analysis presented in this paper, we use 215030 events among those collected from 1 January 2004 to 31 August 2018 with an accumulated exposure of $\mathscr{E}=(60400 \pm 1800) \mathrm{km}^{2} \mathrm{sr}$ yr, $17 \%$ higher than the one used for our previous publication [2].

The estimation of the correction factor, $f\left(E_{i}\right)$, needs knowledge of the resolution in SD energies. Moreover, to account for the migration of the events with energy below the threshold for the saturation of the trigger efficiency, one has to know the trigger efficiency as a function of energy and zenith angle as well as the bias affecting $E_{\mathrm{SD}}$. In fact, when the array is not fully efficient, we 

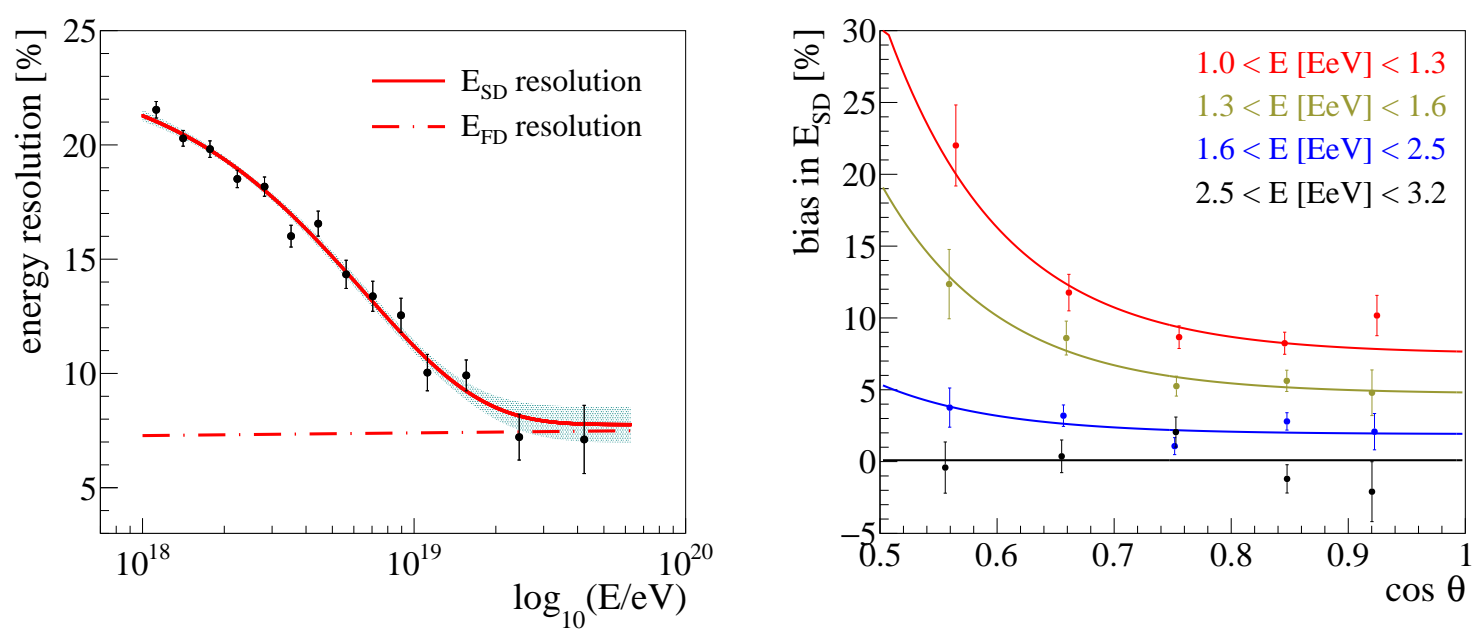

Figure 3: Energy resolution and bias for SD events estimated from hybrid data.

preferably trigger on events with upward fluctuations of muons that lead to higher values of $S_{38}$ and thus to an overestimation of the shower energy. For the measurement presented in this paper all the ingredients needed to calculate $f\left(E_{i}\right)$ are inferred from an analysis of the hybrid data with energy $E_{\mathrm{FD}}>10^{18} \mathrm{eV}$. The trigger efficiency is estimated following the approach described in [11]. It is parametrised with the error function $1 / 2\left\{1+\operatorname{erf}\left[\left(\log _{10} E-p_{0}\right) / p_{1}\right]\right\}$ where $p_{1}=0.373$ and $p_{0}$ is a third degree polynomial in terms of $k=\cos ^{2} \theta\left(p_{0}=\sum_{l}^{[0,3]} y_{l} k^{l}\right)$ with coefficients $\left(y_{0}, y_{1}, y_{2}, y_{3}\right)=$ $(18.63,-3.18,4.38,-1.87)$. The resolution and bias are estimated by studying the distributions of $E_{\mathrm{SD}} / E_{\mathrm{FD}}$ in different energy and zenith angle bins. The distributions are fitted to a Gaussian ratio distribution leaving as free parameters the resolution and bias in $E_{\mathrm{SD}}$ and fixing the resolution in $E_{\mathrm{FD}}$ to about $7.4 \%$ [8]. The results of the analysis are presented in figure 3. The resolution in SD energies is approximatively zenith-angle independent and it is parametrised with the functional form $0.078+0.16 \exp (-0.15 \mathrm{E} / \mathrm{EeV})$. It is estimated with a relative systematic uncertainty ranging from $5 \%$ to $15 \%$ (larger at higher energies). The energy bias below $E_{b}=2.5 \times 10^{18} \mathrm{eV}$ is parametrised with the function $\{0.20+0.59 \exp [-10(\cos \theta-0.5)]\} \log _{10}\left(E_{b} / E\right)$. Above $E_{b}$ the bias is 0 .

The correction factor, $f\left(E_{i}\right)$, is estimated with a "forward folding" technique: we make a fit of $J_{\text {raw }}$ assuming an empirical functional shape for the spectrum defined by a set of free parameters and calculating the bin-to-bin migration matrix due to resolution effects. At the end of the fit $f\left(E_{i}\right)$ is given by the ratio of the input spectrum to the convoluted one. The optimal functional shape can be inferred by looking at the raw energy spectrum. The latter multiplied by $E_{i}^{3}$ is shown in the left panel of figure 4. $J_{\text {raw }}$ shows a dip centered at about $5 \times 10^{18} \mathrm{eV}$ (a feature called the ankle) and an abrupt suppression at the highest energies. A better description of the shape of the spectrum can be obtained by considering the following two functional forms:

$$
\begin{aligned}
J_{12 \Delta} & \propto E^{-\gamma_{1}} \frac{1+\left(E / E_{12}\right)^{\gamma_{1}}}{1+\left(E / E_{12}\right)^{\gamma_{2}}} \frac{1}{1+\left(E / E_{2 \Delta}\right)^{\Delta \gamma}} \\
J_{1234} & \propto E^{-\gamma_{1}} \frac{1+\left(E / E_{12}\right)^{\gamma_{1}}}{1+\left(E / E_{12}\right)^{\gamma_{2}}} \frac{1+\left(E / E_{23}\right)^{\gamma_{2}}}{1+\left(E / E_{23}\right)^{\gamma_{3}}} \frac{1+\left(E / E_{34}\right)^{\gamma_{3}}}{1+\left(E / E_{34}\right)^{\gamma_{4}}}
\end{aligned}
$$


where the first terms common to the two functions define a smooth transition between the two power laws around the ankle. The other terms define the transition at the highest energies: a smooth suppression with fixed curvature with $J_{12 \Delta}$ [2] and two additional transitions between power laws with $J_{1234}$. Thanks to the high quality of the data and the huge statistics of events collected at the Observatory, one can qualitatively appreciate that the data are better described by $J_{1234}$. Therefore we use this function to perform the "forward folding". The raw spectrum and the one corrected for resolution effects are shown in the right panel of figure 4 . They are very similar with a difference that is about $9 \%$ close to $3 \times 10^{18} \mathrm{eV}$, decreasing to below $2 \%$ at $10^{19} \mathrm{eV}$ and slightly increasing up to $5 \%$ at the highest energies. The corrections for resolution effects are small and do not change significantly the shape of the spectrum that is captured by $J_{1234}$. The same outcome is attained if the "forward folding" is done with $J_{12 \Delta}$. Finally, we have verified that the small energy-dependent systematic uncertainties affecting $S(1000)$ [3] do not impact the conclusion that the shape of the spectrum is better described by the $J_{1234}$ function rather than by $J_{12 \Delta}$.

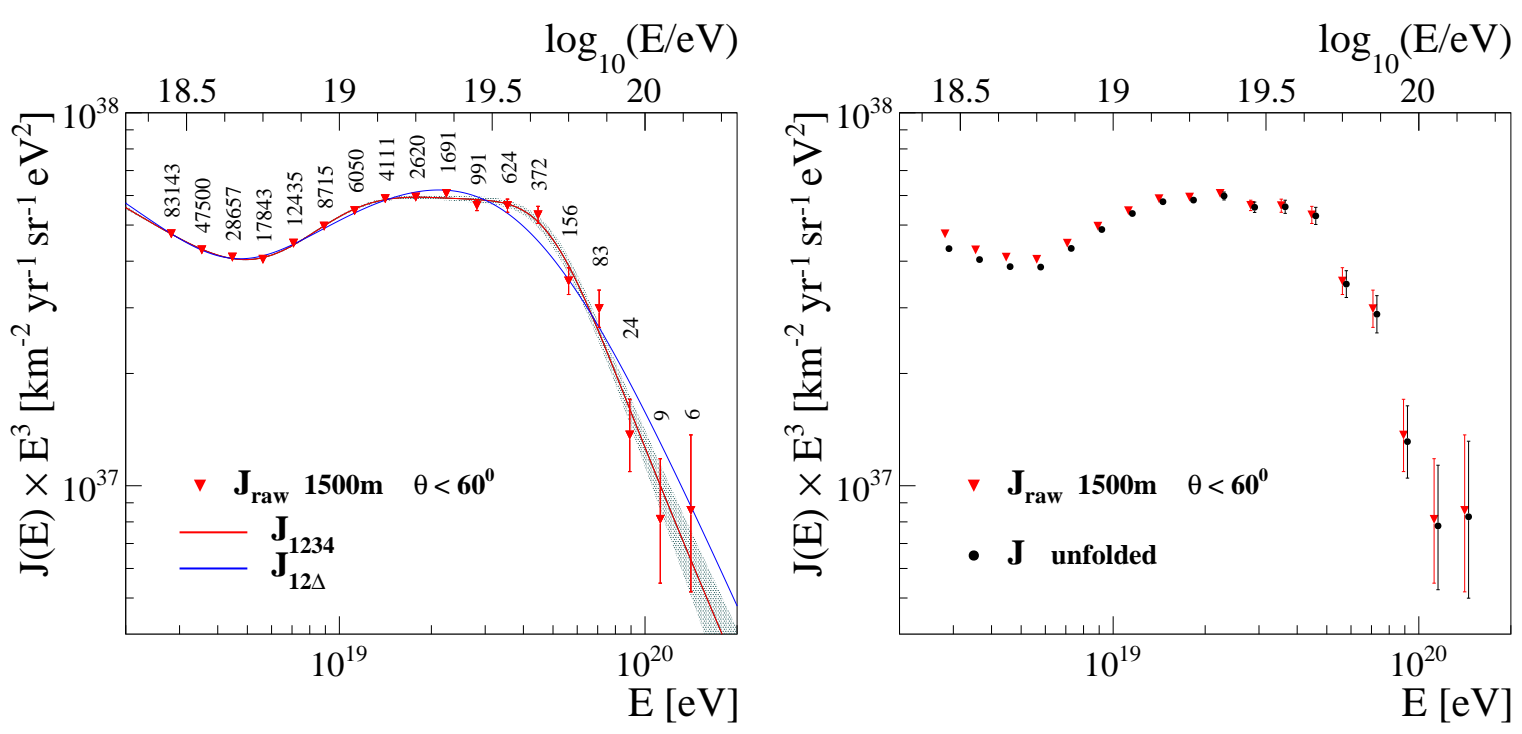

Figure 4: Left panel: raw energy spectrum together with the results of the fit using the two functional forms addressed in the text. Right panel: raw spectrum and the one corrected for resolution effects.

The huge accumulated exposure allows us to measure the spectrum precisely in different declination bands. The results of the studies are reported in [13] and show that the spectrum does not have any significant declination dependence.

\section{Other measurements of the energy spectrum}

The energy spectrum is measured at the Observatory using several independent and complementary data sets. At the highest energies, we increase the SD exposure for events with $\theta<60^{\circ}$ by about $30 \%$ by analysing the events detected at larger zenith angles $\left(60^{\circ}<\theta<80^{\circ}\right)$. In these events, the signals detected by the WCDs are dominated by muons and the energy estimator is given by a normalisation factor of simulated muon density maps that is fitted to the data and calibrated against the FD energies. The spectrum is measured in the energy region where the array is fully efficient $\left(E_{S D}>4 \times 10^{18} \mathrm{eV}\right)$ and using a data-driven approach similar to the one applied to the events with 
Table 1: Relevant parameters of the data samples used to measure the energy spectrum.

\begin{tabular}{|c|c|c|c|c|c|}
\hline & $1500 \mathrm{~m} \theta<60^{\circ}$ & $1500 \mathrm{~m} \theta>60^{\circ}$ & $750 \mathrm{~m}$ & Hybrid & Cherenkov \\
\hline data taking period & $01 / 2004-08 / 2018$ & $01 / 2004-08 / 2018$ & $01 / 2014-08 / 2018$ & $01 / 2007-12 / 2017$ & $06 / 2012-12 / 2015$ \\
\hline exposure $\left[\mathrm{km}^{2} \mathrm{sr} \mathrm{yr}\right]$ & 60426 & 17447 & 105.4 & 2248 at $10^{19} \mathrm{eV}$ & 2.86 at $10^{17} \mathrm{eV}$ \\
\hline number of events & 215030 & 24209 & 569285 & 13655 & 69793 \\
\hline zenith angle range $\left[{ }^{\circ}\right]$ & $0-60$ & $60-80$ & $0-40$ & $0-60$ & $0-85$ \\
\hline energy threshold [eV] & $10^{18.4}$ & $10^{18.6}$ & $10^{17}$ & $10^{18}$ & $10^{16.5}$ \\
\hline \multirow{3}{*}{$\begin{array}{l}\text { energy resolution [\%] } \\
\text { (from low to high E) }\end{array}$} & $18-8$ & $22-10$ & $22-8$ & 7.4 & 18 \\
\hline & & & & & \\
\hline & \multicolumn{5}{|c|}{ calibration parameters } \\
\hline number of events & 3338 & 393 & 1179 & & \\
\hline$A[\mathrm{EeV}]$ & $0.186 \pm 0.003$ & $5.51 \pm 0.07$ & $0.0132 \pm 0.0004$ & & \\
\hline B & $1.031 \pm 0.004$ & $1.04 \pm 0.02$ & $1.006 \pm 0.009$ & & \\
\hline
\end{tabular}
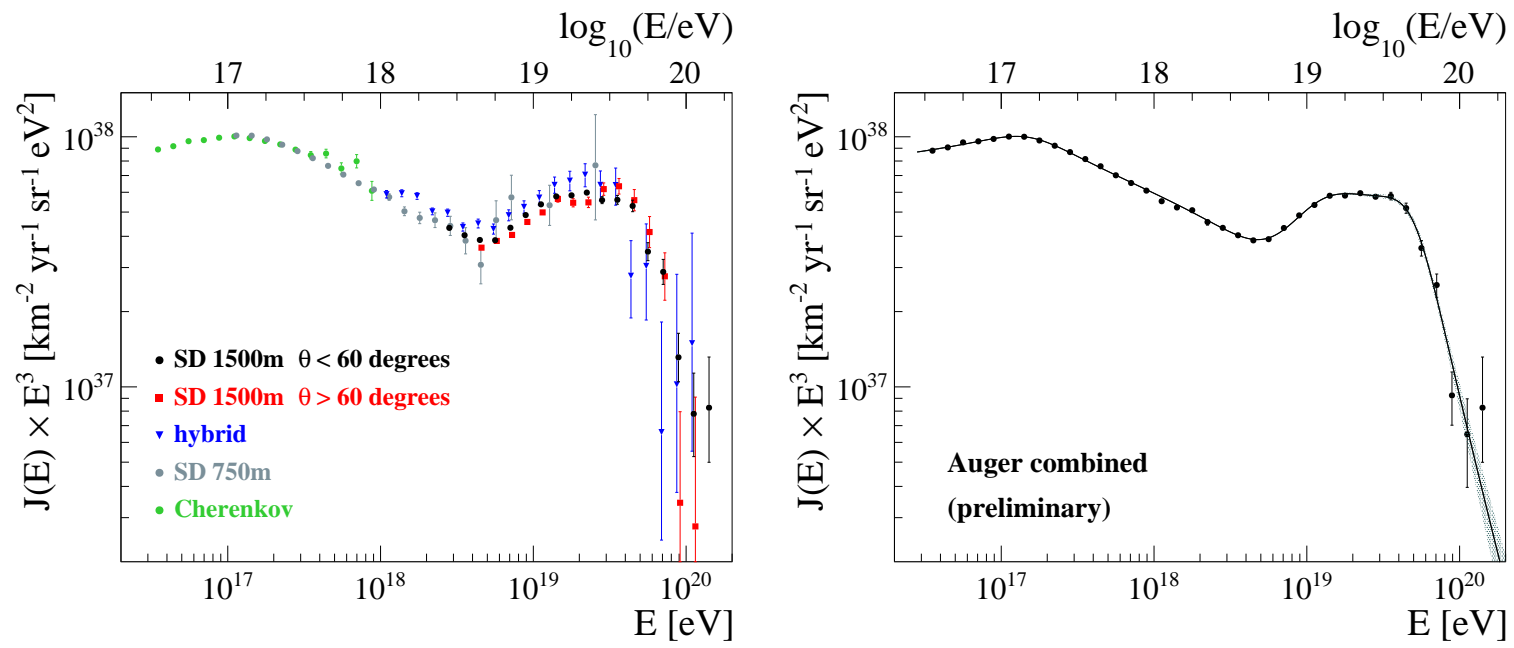

Figure 5: Energy spectra measured using the Pierre Auger Observatory (left) and spectrum obtained combining the different measurements (right).

$\theta<60^{\circ}$ (see also [11]). Another measurement of the spectrum is obtained by analysing the hybrid events detected by the FD simultaneously with at least one WCD. The measurement benefits from the high precision in the FD energy estimation and is made selecting events with energy $>10^{18} \mathrm{eV}$. The exposure is calculated using a full time-dependent simulation of the hybrid events and detector response [12].

The spectrum measurements are extended to lower energies using the $750 \mathrm{~m}$ array. Thanks to the implementation of a new trigger algorithm at the WCD level, in comparison to our previous publication [2], we have been able to lower the energy threshold by half a decade down to $10^{17}$ $\mathrm{eV}$ [14]. This measurement is unique of its kind, similar to the one performed with the $1500 \mathrm{~m}$ array, because it is done with an array in the regime of full trigger efficiency and using a fully datadriven approach. Finally, as pioneered by the Telescope Array [15], for the first time we show the spectrum derived using the events detected by HEAT in which the observed light is dominated by Cherenkov radiation. This allows us to lower the energy threshold to $10^{16.5} \mathrm{eV} \mathrm{[16]} \mathrm{and,} \mathrm{together}$ with the $750 \mathrm{~m}$ spectrum, to precisely study the spectral features around $10^{17} \mathrm{eV}$.

The parameters used to define the various spectra are detailed in table 1 and the measured spectra multiplied by $E_{i}^{3}$ are shown in the left panel of figure 5 . The spectrum obtained by com- 
bining the five measurements is shown in the right panel of figure 5. The combined spectrum is obtained through shifting by $+5 \%$ and $-9 \%$ the normalisations of the $1500 \mathrm{~m} \theta>60^{\circ}$ and the hybrid spectra, respectively, and by $-1 \%$ those both the $750 \mathrm{~m}$ and Cherenkov spectra, while the shift for the $1500 \mathrm{~m} \theta<60^{\circ}$ spectrum is negligible. A fit to the data is performed using an extension of the function (2.3) that includes the smooth change of the spectral index around $10^{17} \mathrm{eV}$

$$
J_{01234} \propto E^{-\gamma_{0}} \frac{1+\left(E / E_{01}\right)^{\gamma_{0}}}{1+\left(E / E_{01}\right)^{\gamma_{1}}} \frac{1+\left(E / E_{12}\right)^{\gamma_{1}}}{1+\left(E / E_{12}\right)^{\gamma_{2}}} \frac{1+\left(E / E_{23}\right)^{\gamma_{2}}}{1+\left(E / E_{23}\right)^{\gamma_{3}}} \frac{1+\left(E / E_{34}\right)^{\gamma_{3}}}{1+\left(E / E_{34}\right)^{\gamma_{4}}} .
$$

The fitted functional form is shown with a black line superimposed to the data. The fitted parameters are: $E_{01}=(0.15 \pm 0.02) \times 10^{18} \mathrm{eV}, E_{12}=(6.2 \pm 0.9) \times 10^{18} \mathrm{eV}, E_{23}=(12 \pm 2) \times 10^{18} \mathrm{eV}$, $E_{34}=(50 \pm 7) \times 10^{18} \mathrm{eV}, \gamma_{0}=2.92 \pm 0.05, \gamma_{1}=3.27 \pm 0.05, \gamma_{2}=2.2 \pm 0.2, \gamma_{3}=3.2 \pm 0.1$ and $\gamma_{4}=5.4 \pm 0.6$, where the errors include the statistical and systematic uncertainties. The data show with high significance two inflection points commonly called the second-knee and the ankle, an indication of a further point of inflection as already addressed in section 2, and the abrupt suppression at the highest energies.

\section{References}

[1] The Pierre Auger Collaboration, Nucl. Instrum. Meth. A 798 (2015) 172.

[2] F. Fenu, for the Pierre Auger Collaboration, Proc. of 35th Int. Cosmic Ray Conf., Bexco, Busan, Korea, PoS(ICRC2017)486.

[3] D. Mockler, for the Pierre Auger Collaboration, these proceedings, PoS(ICRC2019)353.

[4] D. Newton, J. Knapp and A. A. Watson, Astropart. Phys. 26 (2007) 414.

[5] J. Hersil et al., Phys. Rev. Lett. 6 (1961) 22.

[6] The Pierre Auger Collaboration, submitted to PRD (2019).

[7] The Pierre Auger Collaboration, Nucl. Instrum. Meth. A 613 (2010) 29.

[8] B. Dawson, for the Pierre Auger Collaboration, these proceedings, PoS(ICRC2019)231.

[9] V. Verzi, for the Pierre Auger Collaboration, Proc. of 33rd Int. Cosmic Ray Conf., Rio de Janeiro, Brazil (2013) [arXiv:1307.5059].

[10] M. Ave et al., Astropart. Phys. 42 (2013) 90.

[11] The Pierre Auger Collaboration, JCAP 08 (2015) 049.

[12] The Pierre Auger Collaboration, Astropart. Phys. 34 (2011) 368.

[13] O. Deligny, for the Pierre Auger and Telescope Array Collaborations, these proceedings, PoS(ICRC2019)234.

[14] A. Coleman, for the Pierre Auger Collaboration, these proceedings, PoS(ICRC2019)225.

[15] R. U. Abbasi et al., Astrophys. J. 865 (2018) no.1, 74.

[16] V. Novotny, for the Pierre Auger Collaboration, these proceedings, PoS(ICRC2019)374. 